\title{
Osteoblast and Bacterial Culture from Cryopreserved Skull Flap after Craniectomy : Laboratory Study
}

\author{
Tack Geun Cho, M.D., Ph.D., ${ }^{1}$ Suk Hyung Kang, M.D., Ph.D., ${ }^{2}$ Yong Jun Cho, M.D., Ph.D., ${ }^{2}$ Hyuk Jai Choi, M.D., Ph.D., ${ }^{2}$ \\ Jin Pyeong Jeon, M.D., Ph.D., ${ }^{2}$ Jin Seo Yang, M.D. ${ }^{2}$ \\ Department of Neurosurgery, Kangnam Sacred Heart Hospital, Hallym University College of Medicine, Seoul, Korea \\ Department of Neurosurgery, ${ }^{2}$ Chuncheon Sacred Heart Hospital, Hallym University College of Medicine, Chuncheon, Korea
}

Objective : Cranioplasty using a cryopreserved skull flap is a wide spread practice. The most well-known complications of cranioplasty are postoperative surgical infections and bone flap resorption. In order to find biological evidence of cryopreserved cranioplasty, we investigated microorganism contamination of cryopreserved skulls and cultured osteoblasts from cryopreserved skulls.

Methods : Cryopreserved skull flaps of expired patients stored in a bone bank were used. Cryopreserved skulls were packaged in a plastic bag and wrapped with cotton cloth twice. After being crushed by a hammer, cancellous bone between the inner and outer table was obtained. The cancellous bone chips were thawed in a water bath of $30^{\circ} \mathrm{C}$ rapidly. After this, osteoblast culture and general microorganism culture were executed. Osteoblast cultures were done for 3 weeks. Microorganism cultures were done for 72 hours.

Results : A total of 47 cryopreserved skull flaps obtained from craniectomy was enrolled. Of the sample, 11 people were women, and the average age of patients was 55.8 years. Twenty four people had traumatic brain injuries, and 23 people had vascular diseases. Among the patients with traumatic brain injuries, two had fracture compound comminuted depressed. The duration of cryopreservation was, on average, 83.2 months (9 to 161 months). No cultured osteoblast was observed. No microorganisms were cultured.

Conclusion : In this study, neither microorganisms nor osteoblasts were cultured. The biological validity of cryopreserved skulls cranioplasty was considered low. However, the usage of cryopreserved skulls for cranioplasty is worthy of further investigation in the aspect of cost-effectiveness and risk-benefit of post-cranioplasty infection.

Key Words : Decompressive craniectomy · Cranioplst · Cryopreservation · Osteoblast · Cell culture techniques · Bacterial infections.

- Received : January 5, 2017 •Revised : March 24, 2017 •Accepted : May 4, 2017

- Address for reprints : Suk-Hyung Kang, M.D., Ph.D.

Department of Neurosurgery, Chuncheon Sacred Heart Hospital, Hallym University College of Medicine, 77 Sakju-ro, Chuncheon 24253, Korea

Tel : +82-33-240-5173, Fax: +82-33-242-9970, E-mail : nscharimsa@hanmail.net

This is an Open Access article distributed under the terms of the Creative Commons Attribution Non-Commercial License (http://creativecommons.org/licenses/by-nc/4.0) which permits unrestricted non-commercial use, distribution, and reproduction in any medium, provided the original work is properly cited. 


\section{INTRODUCTION}

The most effective surgical method for increased intracranial pressure not responding to medical treatments is craniectomy, and the skull flap obtained from craniectomy is either cryopreserved or banked in a subcutaneous pocket in the patient's abdominal wall ${ }^{3,4,7,18,24,36)}$.

Cranioplasty using a patient's autologous cryopreserved skull flap is a widespread practice across the world. The merits of using cryopreserved skull flaps are its inexpensive cost and cosmetically natural output ${ }^{21)}$. The most well-known complications of cranioplasty are postoperative surgical infections and bone flap resorption ${ }^{33)}$. Bhaskar reported "bone flap nonviability may be a contributing factor" of these complications $^{6}$. For this reason, there are many opportunities to test and see the viability of cryopreserved skulls ${ }^{6,14,19,28)}$. However, biological evidence of cryopreserved auto-bone cranioplasty is obscure.

Cranioplasty with an autologous fresh skull flap leads to very strong bony fusion. The osteogenic, osteoinductive and osteoconductive materials are rich in fresh bone flap. However, whether these three components are present in a cryopreserved skull flap remains uncertain. There are few reports on the osteogenic potential of a cryopreserved skull flap. It has been reported that osteoblasts were not cultured from skull flaps cryopreserved for more than 6 months ${ }^{6}$. There are very few reports regarding the fate of a transplanted cryopreserved skull. Would the cryopreserved skull become a transplanted organ or just be a histocompatible scaffold containing osteoinductive and osteoconductive materials?

The prime objective of this study was to find biological evidence of cranioplasty with autologous cryopreserved skull flaps. The first step was to extract viable cells from cryopreserved skulls. In this study, we tried to extract osteoblasts, an essential cellular component of osteogenesis, from cryopreserved skull flaps. The other objective was to investigate microorganism contamination while thawing the skull, a process that may cause post-cranioplasty infection.

\section{MATERIALS AND METHODS}

The bone flaps from decompressive craniectomy were stored in a freezer with a temperature of $-70^{\circ} \mathrm{C}$. Before cryo- preservation, the bone flaps were packaged in a plastic vinyl bag and packaged two more times with sterile cotton cloths. The skull flaps were collected from expired patients, and this protocol was conducted after receiving deliberations of Institutional Review Board (2015-123).

The cryopreserved bone flap packages were crushed with a hammer and the resulting bone fragments were used. Using sterile surgical instruments, cancellous bone between the inner and outer table was obtained. The cancellous bone chips were put into sterile phosphate buffer saline (PBS) and thawed in a water bath of $30^{\circ} \mathrm{C}$ for 10 minutes. After this thawing process, osteoblasts culture and general microorganism culture from bone chips were executed (Fig. 1A).

We followed bone explant methods as reported by Bhaskar et al. ") "For bone explant cultures, the bone samples were initially rinsed with sterile PBS and transferred to a clean dish containing $2 \mathrm{~mL}$ to $3 \mathrm{~mL}$ of PBS. The bone samples were further diced into pieces measuring approximately $3 \mathrm{~mm}$ in maximal dimensions. After decanting the PBS, the bone chips were transferred to a sterile $50 \mathrm{~mL}$ tube with $15 \mathrm{~mL}$ to $20 \mathrm{~mL}$ of PBS and placed in a vortex mixer for 30 seconds. The supernatant was then decanted and the process repeated to wash off marrow and fat debris. The washed bone fragments were cultured as explants at a density of 0.2-0.6 gm of tissue/100 $\mathrm{mm}$ dish in $10 \mathrm{~mL}$ of Dulbecco's Modified Eagle's medium enriched with $10 \%$ fetal bovine serum, $100 \mathrm{~L} / \mathrm{mL}$ penicillin and $100 \mu \mathrm{g} / \mathrm{mL}$ streptomycin and incubated at $37^{\circ} \mathrm{C}$ in $5 \%$ carbon dioxide $\left(\mathrm{CO}_{2}\right)$. These cultures were maintained undisturbed for 7 days, following which the culture medium was replaced with an equal volume of fresh complete medium, taking care not to dislodge the explants. After 14 days, the medium was changed twice weekly thereafter. This process was repeated and the cultures were subjected to microscopic examination for presence of cell growth at 7 days, 14 days, and 21 days." (Fig. 1B).

To confirm osteoblast culture, alkaline phosphatase (ALP) staining was performed with leukocyte alkaline phosphatase kit (Sigma Aldrich, St Louis, MO, USA). The culture media was aspirated and washed with PBS. Cells were fixed with 0.5 $\mathrm{mL}$ citrate, $1.3 \mathrm{~mL}$ acetone and $0.16 \mathrm{mg}$ formaldehyde solution at room temperature for 1 minute. After fixative removal, the prepared staining solution with a water buffer was added to cover the plate. After covering the plate with foil, the cells were incubated for 15 minutes. The plate was rinsed with PBS 

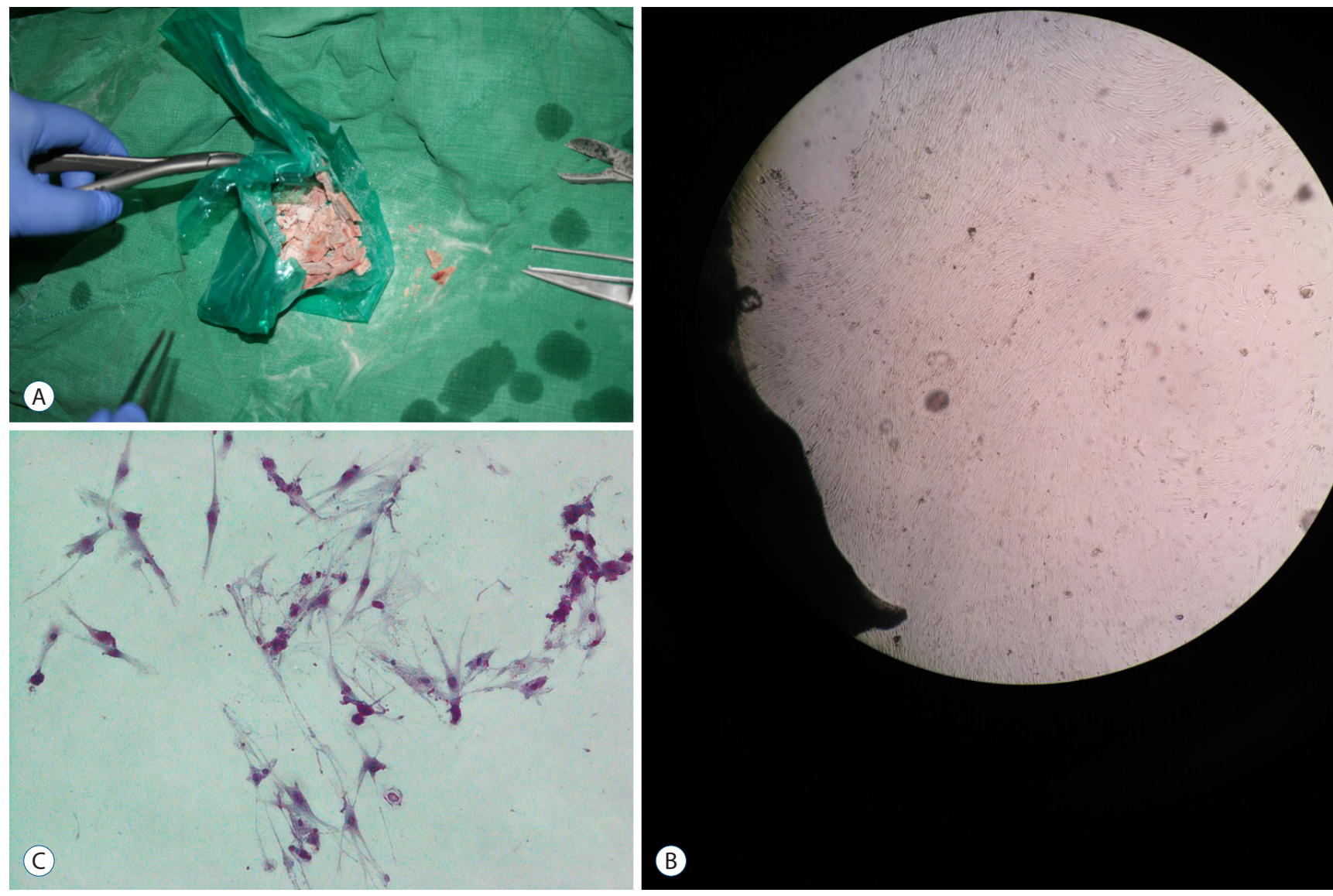

Fig. 1. Osteoblast extraction method from cryopreserved skull. A : The cryopreserved skull flap packages were crushed by hammer and bone fragments were used. Using sterile surgical instrument, cancellous bone between inner and outer table was obtained. B : Low power field polarization microscopic examintaion 21 days after culture showed abundant spindle-like cells around bone chips $(\times 40)$. C : High power field microscopic examination with alkaline phosphatase staining showed purple colored stained cell as described in the Materials and Methods sections (ALP staining, $\times 200$ ).

twice. ALP-positive cells showed purple or red stains under microscopic examination (Fig. 1C).

For microorganism cultures, MacConkey agar plates, Chocolate agar plates and Sabouraud Dextrose agar plates were used in $37^{\circ} \mathrm{C}$. For 72 hours, the colony cultures were observed.

\section{RESULTS}

A total of 47 cryopreserved skull flaps obtained from craniectomy between August 2002 and May 2015 was enrolled. Of the sample, 11 people were women, and the average age of patients was 55.8 years at the time of surgery. Twenty three patients had vascular diseases, and 24 patients had traumatic brain injuries. Among the patients with traumatic brain inju- ries, 2 patients had open fractures. From surgery to thawing, the average cryopreservation period was 83.2 months. The shortest period was 9 months and longest period was 161 months. During 21 days of observation, no cell growth from cryopreserved skull flap was observed. No microorganisms were cultured.

\section{DISCUSSION}

In this study, we attempted to extract osteoblasts and culture the microorganisms from cryopreserved skull flaps obtained from decompressive craniectomy. We used the skull flaps of 47 patients; however, neither osteoblasts nor microorganisms were cultured. 


\section{Cranioplasty and bone flap cryopreservation af- ter decompressive craniectomy}

Decompressive craniectomy is a traditional, classic and evidence-based surgical method for intractable increased intracranial pressure $^{4)}$. Cranioplasty after decompressive craniectomy is an essential and classic surgical method; we can even find evidence of the ancient Incan civilization practicing cranioplasty from historical records. The increase of decompressive craniectomy is leading to an increase in cranioplasty ${ }^{9)}$.

The reason why we should perform cranioplasty after craniectomy is to protect the brain, achieve a natural appearance and prevent sinking skin flap syndrome (or syndrome of the trephined). Furthermore, cranioplasty may improve neuronal function after craniectomy. Many documents report cranioplasty enhances cerebral glucose metabolism, cerebrovascular reserve capacity, postural blood flow regulation and cerebrospinal fluid circulation ${ }^{8,9,12)}$. Clinically, cranioplasty improves cortical perfusion $^{23)}$. In animal study, these phenomena are reproduced $^{17)}$.

How was the skull flap preserved sterilely after craniectomy? There are various methods to sterilize autologous skull flaps for cranioplasty, such as subcutaneous layer placing, cryopreservation or dumping ${ }^{1,2,18,25)}$. The advancements in freezing technology allows cryopreservation to become a more economic and practical method; the most noteworthy devel- opment being the deep-freezer ${ }^{11)}$. Biomechanical studies reveal that the freezing and thawing processes have little effect on the mechanical properties of the human skull ${ }^{35)}$. However, there is not a lot of information on how to cryopreserve skull flaps. There is no protocol for bone flap cryopreservation. According to Bhaskar, 96\% of 25 neurosurgical centers in Australia have been using cryopreserved autologous bone flaps for cranioplasty $^{7)}$. In his report, $88 \%$ of institutions used double or triple bagged under dry, sterile conditions for packing bone flaps. The bone flaps were cryopreserved at between $-18^{\circ} \mathrm{C}$ and $-83^{\circ} \mathrm{C}^{7)}$.

\section{What happens after cranioplasty and why were osteoblasts not extracted in this study?}

The fresh bone flap is usually reinserted immediately after craniotomy. Reinserted bone flaps undergo complex healing processes to fuse with surrounding bones ${ }^{29)}$. Capillary invasion and osteoblast migration from surrounding bones are important for the bone flap's survival. After reinsertion, no blood is supplied to the bone flap, leading to ischemia. The bone flap is surrounded by blood and inflammatory response is initiated. Capillaries from surrounding bone, dura and periosteum infiltrate the reinserted bone. As granulation tissue proliferates, capillaries invade the reinserted bone flap. Through the capillary, primitive progenitor cell migrates and
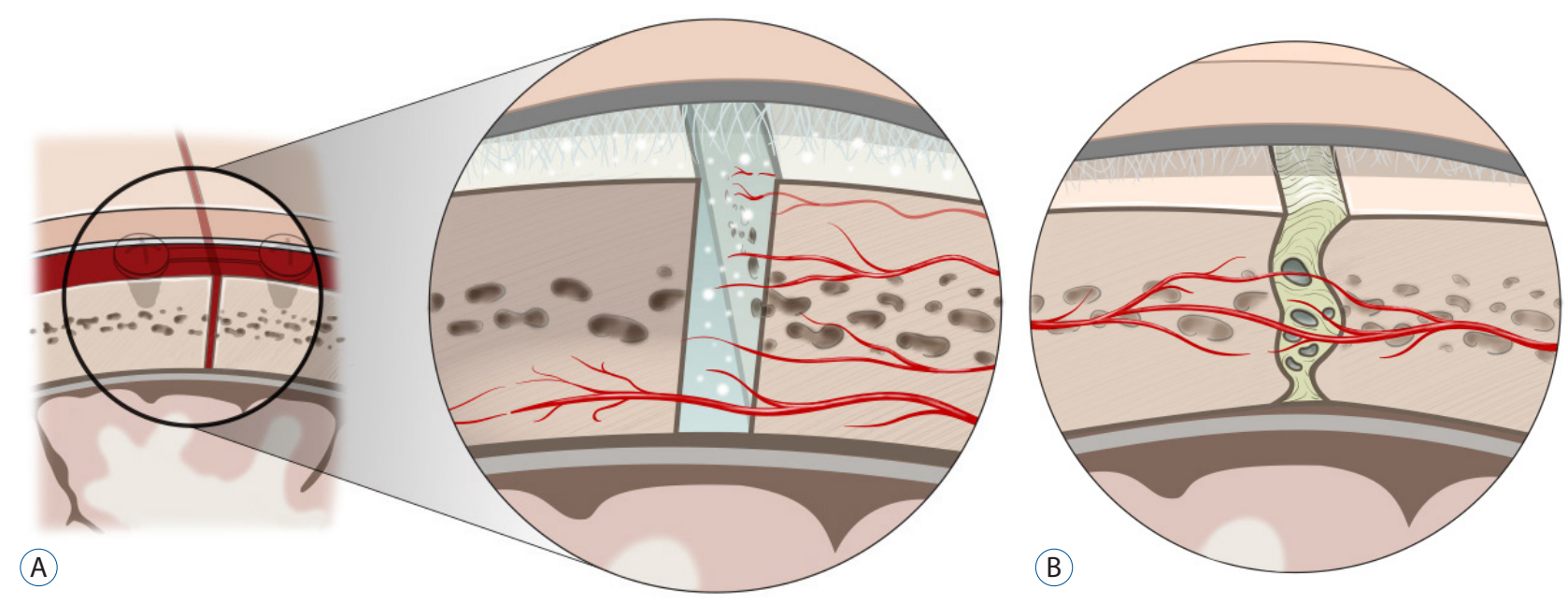

Fig. 2. Schematic figure of bone fusion process after craniotomy. A : Inflammatory phase. The bone flap is surrounded by blood and inflammatory response is initiated. (Top to bottom) Capillaries from surrounding bone, dura and periosteum infiltrate to the transplanted bone. As granulation tissue proliferated, capillaries invade the transplanted bone flap. Through the capillary, primitive progenitor cell migrated and bone remodeling occurred. If this functional contact between the transplanted flap and surrounding bone is poor, re-inserted bone flap would be in ischemic necrosis, and be absorbed. B : Callus formation phase. Cartilage and fibrous tissue is laid down and make new lamellar bone, which is remodeled with osteoclastosteoblast coupling activity to strong bone fusion. 
bone remodeling occurs ${ }^{5}$. Necrotic bone is gradually reabsorbed. If this functional contact between the reinserted flap and surrounding bone is poor, the reinserted flap will be absorbed, partially or totally (Fig. 2) ${ }^{29}$. In an animal study, free skull flaps showed a 50\% reduction in volume after reinser$\operatorname{tion}^{13}$. Similarly, an experimental study on cranioplasty using bone particles also showed that half of the on-lay graft is reabsorbed $^{10)}$.

After decompressive craniectomy, bone flap reinsertions were delayed. Bone flaps were stored in abdominal subcutaneous pockets or were cryopreserved. Oh et al, reported that special freezing techniques were required to maintain osteoblast viability in frozen human iliac cancellous bone ${ }^{24)}$. Simpson reported 3.1\% of osteoblast-like cell cultures from frozen cancellous bones were harvested from femoral head and stored for more than 6 months $^{31}$. However, the osteogenic capacity of skull is markedly deficient than that of long bone ${ }^{29)}$. Furthermore, cell damage occurs during the freezing and thawing procedures ${ }^{24)}$. Some reports recommend bone flap cryopreservation with glycerol, known as cryoprotectant solutions ${ }^{30,34)}$. In another animal study, a specifically programmed freezer is required for cranial bone regeneration from a cryopreserved skull ${ }^{19)}$. We recommend that operators reduce the gap between cryopreserved flaps and viable skulls to encourage osteogenic progenitor cell migration to reduce bone resorption. Wider contact surface would be promoting osteogenic progenitor cell migration from the host skull to the cryopreserved flap, which has rich histocompatible osteoinductive and osteoconductive materials. We think that periosteum preservation during craniectomy and cranioplasty is also important to promote bone fusion between the cryopreserved flap and the host skull.

We believe there were several reasons why osteoblasts were not extracted in this study. The average age of enrolled patients was 55.8 years ( $\min 20, \max 87$ ), having a relatively low regeneration power. The cryopreservation period was longer than 6 months. There was little cancellous bone marrow in the skull flap rather than femoral heads ${ }^{31}$. There were no special cryopreservation protocols such as cryopreservation time interval from bone flap extraction to cryopreservation, deep freezing technique, duration, and cryoprotectant use.

In this study, no culture of an osteoblast was the same as in the Bhaskar's study ${ }^{6}$, and we think it is meaningful to reproduce the existing negative results. And Bhaskar's study ${ }^{6}$ had
27 samples, but the number of samples in this study was 47 , and the cryopreservation was frozen at -30 degrees in Bhaskar's paper ${ }^{6}$, but we kept it at -70 degrees. In addition, this paper differs from previous papers in that it performed not only osteoblast cell culture but also bacterial culture. The use of non-viable tissue as well as contamination in relation to postoperative infection may be a bigger problem. However, after reviewing published papers, we think that it is difficult to conclude that the cryopreserved skull is a non-viable tissue. Therefore, it would be meaningful to suggest that the bacterium was not cultured in the cryopreserved skull.

\section{Economics of cryopreserved skull flap ${ }^{22)}$}

Cryopreserved skull flaps have osteoinductive and osteoconductive characters biologically. If we could extract osteoblasts from cryopreserved skull flaps, we can prove this is an ideal material for cranioplasty. We think cranioplasty with cryopreserved autologous skulls is valuable in two aspects.

First, biologically cryopreserved skull flaps are the best scaffold for autologous osteoblasts. In the concept of tissue-engineered constructs, cryopreserved skull flaps are filled with osteoconductive and osteoinductive materials, which were completely matched with the recipient immunologically ${ }^{20)}$. Cryopreserved skulls are the most ideal substitute for cranioplasty.

Seconds, the cryopreserved skull flaps are economic. There are many reports about new substitute for cranioplasty, recently ${ }^{21,22,26,27,32)}$. The difference of the clinical outcome between synthetic materials and autologous bone graft in cranioplasty is very small. Some authors report lower complication with synthetic materials ${ }^{7,25-28)}$. Many researchers emphasize the economic merit of cranioplasty with autologous cranioplas$t^{15,21,22)}$. However, opposing viewpoints do exist, such as reports from USA \& Canada that report the total cumulative cost, including possible complication treatments between titanium cranioplasty and autologous cranioplasty, was not significantly different ${ }^{16,34)}$. It is only natural that the surgical cost of cranioplasty is different in each country because the medical service systems are different. In the Republic of Korea, cranioplasty using synthetic materials is three times more expensive than cranioplasty using a cryopreserved skull. Thus, cranioplasty using cryopreserved skull flaps is more economic than synthetic materials in Republic of Korea.

In this study, the minimum duration of cryopreservation 
was 9 months. If there were bone flaps cryopreserved for periods shorter than 6 months, the result may have been different. However, we maintain that the possibility of osteoblast extraction would still be very low, even if the cryopreservation period was shorter than 6 months because there was no report about osteoblast explant from cryopreserved skull in human study. Unfortunately, we could not find any reports on the regenerative potential of skulls. However, we know that the skull has an inner and outer cortical table, and the cancellous bone between them is very small. As patients get older, the hematopoietic potentials of bone marrow decrease. Most patients are adults, not children.

\section{CONCLUSION}

In this study, neither microorganisms nor osteoblasts were cultured. On the positive side, no evidence of frozen skull contamination, even during the thawing process, was found. No cultured osteoblasts were found in the cryopreserved skulls. The biological validity of using cryopreserved skulls for cranioplasty was considered low. However, the usage of cryopreserved skulls for cranioplasty is worthy of further investigation in the aspect of cost-effectiveness and risk-benefit of post-cranioplasty infection.

\section{- Acknowledgements}

This study was supported by BioGreen21 Program (PJ01121401) of Rural Development Administration of ROK. This work was supported by a grant from Hallym University Medical Center Research Fund (HURF 2015-42).

We are thankful to Chi Hern Lee and Noar Kim who provided expertise that greatly assisted the research.

\section{References}

1. Abbott KH : Use of frozen cranial bone flaps for autogenous and homologous grafts in cranioplasty and spinal interbody fusion. J Neurosurg $10: 380-388,1953$

2. Asano $Y$, Ryuke $Y$, Hasuo $M$, Simosawa $S$ : Cranioplasty using cryopreserved autogenous bone. No To Shinkei 45 : 1145-1150, 1993

3. Baldo S, Tacconi L : Effectiveness and safety of subcutaneous abdominal preservation of autologous bone flap after decompressive craniectomy: a prospective pilot study. World Neurosurg $73: 552-556,2010$

4. Barthelemy EJ, Melis M, Gordon E, Ullman JS, Germano IM : Decompres- sive craniectomy for severe traumatic brain injury: a systematic review. World Neurosurg 88 : 411-420, 2016

5. Benzel EC, Gilbertson L, Mericle RA : Enhancing spinal fusion. Clin Neurosurg $55: 63-71,2008$

6. Bhaskar IP, Yusheng L, Zheng M, Lee GY : Autogenous skull flaps stored frozen for more than 6 months: do they remain viable? J Clin Neurosci 18 : 1690-1693, 2011

7. Bhaskar IP, Zaw NN, Zheng M, Lee GY : Bone flap storage following craniectomy: a survey of practices in major Australian neurosurgical centres. ANZ J Surg 81 : 137-141, 2011

8. Carvi Y Nievas MN, Höllerhage HG : Early combined cranioplasty and programmable shunt in patients with skull bone defects and CSFcirculation disorders. Neurol Res 28 : 139-144, 2006

9. Chaturvedi J, Botta R, Prabhuraj AR, Shukla D, Bhat DI, Devi BI : Complications of cranioplasty after decompressive craniectomy for traumatic brain injury. Br J Neurosurg 30 : 264-268, 2016

10. Clune JE, Mulliken JB, Glowacki J, Arany PR, Kulungowski AM, Rogers GF, et al. : Autologous cranial particulate bone graft: an experimental study of onlay cranioplasty. J Craniofac Surg 22 : 319-323, 2011

11. Elliott $H$, Scott $H$ : The bone-bank in neurosurgery. Br J Surg 39 : 31 34, 1951

12. Erdogan E, Düz B, Kocaoglu M, Izci Y, Sirin S, Timurkaynak E : The effect of cranioplasty on cerebral hemodynamics: evaluation with transcranial Doppler sonography. Neurology India 51 : 479-481, 2003

13. Fukuta K, Har-Shai Y, Collares MV, Herschman BR, Persiani RJ, Jackson IT : The viability of revascularized calvarial bone graft in a pig model. Ann Plas Surg 29 : 136-142, 1992

14. Georgiou K, Fan C, Ng Y, Shandala T, King T, Scherer M, et al. : Do cryopreserved autogenous cranial bone flaps remain viable at cranioplasty? Bone 47 : S387-S388, 2010

15. Hayward RD : Cranioplasty: don't forget the patient's own bone is cheaper than titanium. Br J Neurosurg 13 : 490-491, 1999

16. Honeybul S, Morrison DA, Ho KM, Lind CR, Geelhoed E : A randomized controlled trial comparing autologous cranioplasty with custom-made titanium cranioplasty. J Neurosurg $126: 81-90,2017$

17. Hwang G, Oh CW, Han JH, Kim CY, Kwon OK, Park SQ, et al. : Decompressive craniectomy for malignant middle cerebral infarction. Korean J Cerebrovasc Surg 11 : 49-54, 2009

18. Joaquim $A F$, Mattos JP, Neto FC, Lopes $A$, de Oliveira E : Bone flap management in neurosurgery. Revista Neurociencias 17 : 133-137, 2009

19. Kaku M, Koseki H, Kojima S, Sumi H, Shikata H, Kojima S, et al. : Crania bone regeneration after cranioplasty using cryopreserved autogenous bone by a programmed freezer with a magnetic field in rats. Cryo Letters $35: 451-461,2014$

20. Kuleshova LL, Gouk SS, Hutmacher DW : Vitrification as a prospect for cryopreservation of tissue-engineered constructs. Biomaterials 28 : 1585-1596, 2007

21. Lemée JM, Petit $D$, Splingard M, Menei P : Autologous bone flap versus hydroxyapatite prosthesis in first intention in secondary cranioplasty after decompressive craniectomy: a French medico-economical study. Neurochirurgie 59 : 60-63, 2013 
22. Lethaus B, Bloebaum $M$, Koper $D$, Poort-Ter Laak M, Kessler $P$ : Interval cranioplasty with patient-specific implants and autogenous bone grafts-success and cost analysis. J Craniomaxillofac Surg 42 : 1948-1951, 2014

23. Mah JK, Kass RA : The impact of cranioplasty on cerebral blood flow and its correlation with clinical outcome in patients underwent decompressive craniectomy. Asian J Neurosurg 11 : 15-21, 2016

24. Oh JH, Zöller JE, Kübler $A$ : A new bone banking technique to maintain osteoblast viability in frozen human iliac cancellous bone. Cryobiology 44 : 279-287, 2002

25. Osawa M, Hara H, Ichinose $Y$, Koyama T, Kobayashi S, Sugita Y : Cranioplasty with a frozen and autoclaved bone flap. Acta Neurochir (Wien) $102: 38-41,1990$

26. Piitulainen JM, Kauko T, Aitasalo KM, Vuorinen V, Vallittu PK, Posti JP : Outcomes of cranioplasty with synthetic materials and autologous bone grafts. World Neurosurg 83 : 708-714, 2015

27. Plum AW, Tatum SA : A comparison between autograft alone, bone cement, and demineralized bone matrix in cranioplasty. Laryngoscope 125 : 1322-1327, 2015

28. Prolo DJ, Burres KP, McLaughlin WT, Christensen AH : Autogenous skull cranioplasty: fresh and preserved (frozen), with consideration of the cellular response. Neurosurgery $4: 18-29,1979$

29. Shaffrey ME, Persing JA, Shaffrey $\mathrm{Cl}$, Delashaw JB, Jane HA : Management of cranial defect, in Apuzzo MLJ (ed) : Brain Surgery : complication avoidance and management. London : Churchill Livingstone
Inc., 1993, pp1373-1400

30. Shimizu S, Morikawa A, Kuga Y, Mouri G, Murata T : Cranioplasty using autogenous bone cryopreserved with dimethylsulfoxide (DMSO). No Shinkei Geka 30 : 479-485, 2002

31. Simpson D, Kakarala G, Hampson K, Steele N, Ashton B : Viable cells survive in fresh frozen human bone allografts. Acta Orthop $78: 26$ 30, 2007

32. Stefini R, Zanotti B, Nataloni A, Martinetti R, Scafuto M, Colasurdo M, et al. : The efficacy of custom-made porous hydroxyapatite prostheses for cranioplasty: evaluation of postmarketing data on 2697 patients. J Appl Biomater Funct Mater 13 : e136-e144, 2015

33. Sundseth J, Sundseth A, Berg-Johnsen J, Sorteberg W, Lindegaard KF : Cranioplasty with autologous cryopreserved bone after decompressive craniectomy: complications and risk factors for developing surgical site infection. Acta Neurochir (Wien) $156:$ 805-811; discussion 811, 2014

34. Takeuchi H, Higashino Y, Hosoda T, Yamada S, Arishima H, Kodera T, et al. : Long-term follow-up of cryopreservation with glycerol of autologous bone flaps for cranioplasty after decompressive craniectomy. Acta Neurochir (Wien) $158:$ 571-575, 2016

35. Torimitsu S, Nishida Y, Takano T, Koizumi Y, Hayakawa M, Yajima D, et al. : Effects of the freezing and thawing process on biomechanical properties of the human skull. Leg Med (Tokyo) $16:$ 102-105, 2014

36. Wang JW, Li JP, Song YL, Tan K, Wang Y, Li T, et al. : Decompressive craniectomy in neurocritical care. J Clin Neurosci 27 : 1-7, 2016 